\title{
Dental Tissues As Forensic Tool In Gender Determination
}

\author{
Gouri $\mathrm{P}^{1}$, Pavan G. Kulkarni ${ }^{2}$, Shyam Prasad Reddy $\mathrm{D}^{3}$, Keerthi $\mathrm{M}^{4}$
}

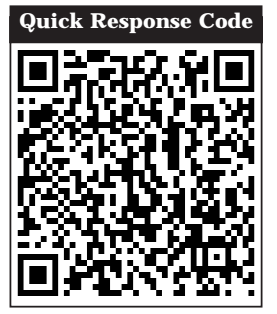

doi: $10.5866 / 2016.8 .10037$

1Postgraduate

${ }^{2}$ Reader

${ }^{3}$ Reader

${ }^{4}$ Senior lecturer

Department of Oral and Maxillofacial Pathology,

Kamineni Institute of Dental Sciences,

Narketpally, Nalgonda Dist, India.

\section{Article Info:}

Received: J anuary 13, 2016

Review Completed: February 12, 2016

Accepted: March 11, 2016

Available Online: May, 2016 (www.nacd.in)

(C) NAD, 2016 - All rights reserved

\section{Email for correspondence:}

gouri.pupala@gmail.com

\section{Introduction:}

Forensic dentistry is an important branch of forensic medicine which contributes immensely in solving difficult criminal cases and also in identification of individuals in mass disasters. In recent times, the world has experienced many mass disasters like acts of terrorism, bombings, earthquakes, hurricanes, tsunami, railway accidents, air crashes and other transportation mishaps. In all these circumstances, as the bodies are skeletonized, decomposed, burned, charred or destroyed beyond visual recognition exact identification of an individual becomes difficult. As teeth and jaws resist extreme temperature

\begin{abstract}
:
Forensic odontology plays an important role in human identification by using dental evidence. Characteristics of teeth remain unchanged even after exposure to extreme environmental conditions for longer duration, which maketeeth as an excellent material for forensic investigations. One of the most important steps in the process of human identification is gender determination. In some situations where the bodies are mutilated beyond recognition, dental remains help in establishing the gender of such victims. This article reviews the importance of the role of dentition in gender determination using clinical methods like measuring the mesiodistal and buccolingual dimensions of the teeth for differentiating sex, importance of permanent canine teeth and their dimorphism, morphological features of the tooth crown or root, microscopic study of $X$ and $Y$ chromosomes and useful ness of genetic markers along with advanced techniques like polymerase chain reaction (PCR) in gender determination.
\end{abstract}

Key words: Forensic odontology, dental tissues, gender determination. 
using skeletal remains, forensic experts can help to determine the gender of the remains by using various features of teeth, like morphology, crown size, root lengths etc., which are characteristic for males and females. Advanced techniques like PCR amplification will assist in determining the gender of the remains more accurately. ${ }^{4}$

\section{Classification of methods used for sex determination:}
I. Visual method or clinical method
II. Microscopic methods
III. Advanced methods

\section{Visual method or clinical methods}

Differences between genders with respect to:
a. Tooth size
b. Root length and crown diameter
c. Using canine diamorphism
d. Tooth morphology and sexing
e. Dental index
f. Odontometric differences
II. Microscopic methods
a. Sex determination using Barr bodies

\section{Advanced methods}

a. Sex determination using Polymerase Chain Reaction (PCR)

b. Sex determination using enamel protein. ${ }^{5}$

\section{Visual methods/ clinical methods:}

a) Size of the tooth: In young individuals where skeletal and secondary sexual characters have not yet devel oped, teeth may be used for gender determination by measuring their mesiodistal and buccolingual dimensions. Significant differences between male and female permanent and deciduous tooth crown dimensions are shown in various studies. It has to be noted that tooth size or odontometrics, is under considerable influence of the environment, hence such measurements are, population specific and therefore do not apply to the world. Amongst teeth, mandibular canines show greatest dimensional difference with larger teeth in males than in females. Premolars, first and second molars, as well as maxillary incisors, also show differences. ${ }^{4}$

b) Root length and crown diameter: Morphological features of the tooth crown or root is due to difference in thicknesses of the enamel which can differentiate between men and women. These features are neither permanent nor al ways reliable, and can be population-specific. $80 \%$ accuracy was observed for gender determination on mandibular permanent teeth by measuring root length and crown diameters using optical scanner and radiogrammetric measurements. ${ }^{4}$

c) Canine dimorphism: Permanent canine teeth and their intercanine distance contribute to gender determination by dimorphism. Various theories have been proposed to explain canine dimorphism. First theory according to Moss, states that, greater thickness of enamel in males due to long period of amelogenesis and slower rate of maturation of teeth than in females, whereas second theory states that, $Y$ chromosomes cause a slow maturation rate of enamel in males. The dimensions of canine teeth have been studied by several methods, including Fourier analysis (Minzuno, 1990), Moire topography (Suzuki et al., 1984) and the measurement of linear dimensions such as mesiodistal width, buccolingual width and incisocervical height. ${ }^{4}$

d) Tooth morphology and sexing: Deflecting wrinkle is the most common morphological characteristic of the tooth which differentiates men from women. Deflecting wrinkle represents a variation of the medial ridge on the mesio-lingual cusp of the first lower molar, wherein the ridge deflects towards the disto-lingual cusp. The existence of this wrinkle is a feature that only appears only in men. Among the acquired morphological characteristics, which differentiate men from women, tooth abrasion is the most important. Due to stronger masticatory muscles and greater masticatory forces, men experience more tooth wear than women, which may lead to a lowered tooth crown height. Distal accessory ridge, a nonmetric feature on the canine is the most sexually dimorphic crown trait in the human dentition, with males showing significantly higher frequencies and more pronounced expression than females. ${ }^{4}$

e) Dental index: In addition to absolute tooth size, tooth proportions have been suggested for gender differentiation. Aitchison presented the "incisor index" (Ii), which is calculated by the formula: I i =[MDI2/MDI 1] ×100, where MDI 2 is the maximum mesiodistal diameter of the maxillary lateral incisor and MDII is the maximum mesiodistal diameter of the central incisor. This index is higher in males, confirming the suggestion of Schrantz and Bartha that the lateral incisor is distinctly smaller than the central incisor in females. 
Another index, the "mandibular canine index" proposed by Rao et al associates have given an accurate indication of gender in the Indian population. Using the mesiodistal (m-d) dimension of the mandibular canines, these researchers obtained the formula:

[(Mean m-d canine dimension + (Mean m-d canine dimension in female + standard deviation [SD]) in males • SD)]/2.

The value obtained using this formula was 7.1, i.e., $7.1 \mathrm{~mm}$ is the maximum possible mesiodistal dimension of mandibular canines in females. The same dimension is greater in males. The success rate of determining sex using the above formula was close to $89 \%$.

f) Odontometric differences: Greater genetic expression explained the odontometric difference between males and females. Lakhanpal et al., reported that males have larger teeth than females and stated that buccolingual dimension is a good gender predictor than mesiodistal dimension. Due to the difficulty in measuring the mesiodistal dimensions due to close proximal contacts, there may be a discrepancy in its measurement. Hence both the dimensions should be considered for gender determination for better and more reliable results.

Though visual or clinical methods are simple and rapid methods for gender determination, they provide only minor details and they can act only as adjuncts to other methods and are not highly reliable. Reliability of visual method is compromised due to factors like regressive alterations of teeth, aging, defects in enamel and dentin formation due to syndrome association, nutritional and environmental causes and so on. Clinical method is useful when other methods of gender prediction cannot be performed. ${ }^{4,5}$

\section{Microscopic methods}

a) Barr bodies: Gender determination can also be done by the study of $X$ and $Y$ chromosomes in the cells that are not undergoing active division. Presence or absence of $X$ chromosome can be studied from buccal smears, skin biopsy, blood, cartilage, hair root sheath, and tooth pulp. After death, it persists for variable periods depending upon the humidity and temperature in which tissue has remained. $X$ chromatin and intra-nuclear structure is also known as Barr body as it was first discovered by Barr et al. It is present as a mass usually lying against the nuclear membrane in females. Duffy et al. have shown that Barr bodies and $F$ bodies of $Y$ chromosomes are preserved in dehydrated pulp tissues up to 1-year and pulp tissues retain diagnostic characteristics when heated up to 100C for 1 h. ${ }^{4}$

\section{Advanced methods:}

Role of deoxyribonucleic acid (DNA) in dental identifications: Because of the resistant nature of dental tissues to environmental assaults, such as incineration, immersion, trauma, mutilation, and decomposition, teeth represent an excellent source of DNA. When conventional dental identification methods fail, this biological material can provide the necessary link to prove identity. ${ }^{6,7}$ DNA preserved in and extracted from the teeth of an unidentified individual can be used to determine the gender of an individual and also can be used by comparing a known antemortem sample like stored blood, hairbrush, clothing, cervical smear, biopsy, etc or to a parent or sibling which helps in human identity. 6,8

Genomic and mitochondrial DNA in forensic dentistry: The genomic DNA is found in the nucleus of each cell in the human body and represents a reliable source for most forensic applications. The teeth are an excellent source of genomic DNA as, body tissues have decomposed, the structures of the enamel, dentin and pulp complex persist for longer durations.

Mitochondrial DNA is another type of material that can be used for human identification. I ts main advantage is the high number of copies per cell (from hundreds to thousands of organelles). When the extracted DNA samples are too small or degraded, such as those obtained from skeletonized tissues, the likelihood of obtaining a DNA profile from mitochondrial DNA is higher than that with any marker found in genomic DNA, but the disadvantage is that mitochondrial DNA is exclusively matrilineal and hence less informative and can be used for comparision of DNA with parent, sibling or relatives, but not appropriate for gender determination. ${ }^{10}$

a) Polymerase chain reaction: Polymerase chain reaction (PCR) is a method of amplifying small quantities of relatively short target sequences of DNA using sequence-specific oligonucleotide primers and thermo-stable Taq DNA polymerase. In situations where there are only few teeth are present or missing dental records, when there is no enough information to identify the person, or when the teeth are subjected to environmental insults the 
dental pulp enclosed by the hard tissue is not influenced by temperature, unlikethe buccal mucous membrane, saliva, and calculus, which serves as a valuable source of genetic information. ${ }^{4,9}$

b) Enamel matrix proteins and forensic markers for sex identification: The ability to determinethe gender of an individual based on DNA evidence can be crucial in instances such as identification of victims of mass disaster, missing persons investigations, and sexual assault cases. As legal gender and chromosomal sex do correspond in the majority of cases, methods for accurate determination of chromosomal sex do and will continue to have widespread use in forensic investigations. $^{11}$

Amelogenin or AMEL is a major matrix proteins found in human enamel belongs to a family of extracellular matrix proteins. It has a different signature (or size and pattern of the nucleotide sequence) in male and female enamel. The AMEL gene that encodes for female amelogenin is located on the distal short arm of $X$ chromosome (AMELX) in p22.1-p22.3 region and AMEL gene that encodes for male amelogenin is located near the centromere of $Y$ chromosome (AMELY) at p11.2 region. Hence, females have two identical AMEL genes or alleles, whereas males have two different $A M E L$ genes. This can be used to determine the gender of the remains with even very small samples of DNA. ${ }^{5,11}$

Sex-determining region $Y$ (SRY) is located on the $Y$ chromosome at p11.31 within a $35 \mathrm{~kb}$ sexdetermining region of the $Y$ chromosome adjacent to the pseudoautosomal region. SRY encodes a transcription factor that is a member of the high mobility group (HMG)-box family of DNA binding proteins and contains the conserved DNA-binding motif indicative of HMG proteins. The SRY genehas been shown to be integral to the development of male reproductive organs. It is the only sex typing marker currently in use whose gene products directly affect biological sex development. The strong association of the products of SRY with male phenotype suggests that SRY is the most accurate available marker for prediction of male appearance and is often used as a confirmatory test when an amelogenin test produces unexpected results. ${ }^{11}$

Both microscopic methods and advanced methods arehighly reliablein gender determination using the dentition. The use of microscopic and advanced methods in gender prediction is expensive, time-consuming, laborious technique of DNA isolation when compared with clinical methods of gender determination. In addition, there are common problems with DNA analysis from human remains due to decomposition and exposure to environmental factors such as high temperatures, humidity, and many organic compounds may result in DNA degradation. Hence, the role of clinical, microscopic and advanced method is equally important in gender prediction when anyone of the method is not applicable due to inevitable circumstances. ${ }^{5}$

\section{Conclusion:}

Forensic dentistry plays a major role in the identification of those individuals who cannot be identified visually or by other means. Principal advantage of dental evidence is that, like other hard tissues, it is often preserved after death and teeth are the most durable parts in the body which can survive virtually intact for longer durations after other soft tissue and skeletal tissue have been destroyed by decay or incineration. Thus, dental tissues play a major role in identifying the gender of an individual.

\section{References:}

1. Manjunath BC, Chandrashekar BR., Melkundi Mahesh et al. DNA Profiling and forensic dentistry - A review of the recent concepts and trends. J Forensic Leg Med 2011; 18:191-7.

2. Singal K. Forensic Dentistry as a Tool for Identification. Austin J Forensic Sci Criminol 2015; 2(5):1-4.

3. Anoop K Verma, Sachil Kumar, Shiuli Rathore et al. Role of dental expert in forensic odontology. Nat J Maxillofacial Surgery 2014; 5(1):2-5.

4. J ayapal Dinakaran, Thayalan Dineshkumar, Gunasekaran Nandhini et al. Gender determination using dentition. SRM J Res Dental Sci 2015; 6(1):29-34.

5. Chalishazar Monali, Panja Pritam, Modi Tapan et al.Gender Determination: A view of forensic odontologist. Ind J Forensic Med Path 2011; 4(4):147-51.

6. Pretty A and Sweet D. A look at forensic dentistry-Part 1: The role of teeth in the determination of human identity. Bri dent j 2001; 190(7):359-66.

7. Nitai debnath, Renu gupta, Rajesh singh nongthombam et al. Forensic odontology. J Med Society 2016; 30(1):20-3.

8. Saranya $\mathrm{V}$, Malathi N. Forensic odontology- A brief review. Sri Ramachandra J Med 2014; 7(2):22-8.

9. Rahman MT, Uddin MS, Sultana R et al. Polymerase chain reaction (PCR) - A short review. AKMMC J 2013; 4(1):30-6.

10. Ricardo Henrique Alves da silvia, Arsenio sales-peres, Rogerio Nogueira de oliveira. Use of DNA technology in forensic dentistry. J Appl Oral Sci 2007; 15(3):156-61.

11. Erin Butler and Richard Li. Genetic markers for sex identification in forensic DNA analysis. J Forensic Investigation 2014;2(3):1-10. 